\title{
Electronic signature of the vacancy ordering in $\mathrm{NbO}\left(\mathrm{Nb}_{3} \mathrm{O}_{3}\right)$
}

\author{
A. K. Efimenko, ${ }^{1}$ N. Hollmann,,${ }^{1}$ K. Hoefer,${ }^{1}$ J. Weinen, ${ }^{1}$ D. Takegami,${ }^{1}$ K. K. Wolff, ${ }^{1}$ S. \\ G. Altendorf, ${ }^{1}$ Z. Hu, ${ }^{1}$ A. D. Rata,${ }^{1}$ A. C. Komarek, ${ }^{1}$ A. A. Nugroho,${ }^{2}$ Y. F. Liao, ${ }^{3}$ K.-D. \\ Tsuei, ${ }^{3}$ H. H. Hsieh, ${ }^{4}$ H.-J. Lin,${ }^{3}$ C. T. Chen, ${ }^{3}$ L. H. Tjeng,, , 尚 and D. Kasinathan ${ }^{1, \dagger}$ \\ ${ }^{1}$ Max Planck Institute for Chemical Physics of Solids, Noethnitzer Str. 40, 01187 Dresden, Germany \\ ${ }^{2}$ Insitiut Teknologi Bandung, Jl. Ganesha 10, 40132 Bandung, Indonesia \\ ${ }^{3}$ National Synchrotron Radiation Research Center (NSRRC), 101 Hsin-Ann Road, 30077 Hsinchu, Taiwan \\ ${ }^{4}$ Chung Cheng Institute of Technology, National Defense University, Taoyuan 335, Taiwan
}

(Dated: March 6, 2018)

\begin{abstract}
We investigated the electronic structure of the vacancy-ordered $4 d$-transition metal monoxide $\mathrm{NbO}\left(\mathrm{Nb}_{3} \mathrm{O}_{3}\right)$ using angle-integrated soft- and hard-x-ray photoelectron spectroscopy as well as ultra-violet angle-resolved photoelectron spectroscopy. We found that density-functional-based band structure calculations can describe the spectral features accurately provided that self-interaction effects are taken into account. In the angle-resolved spectra we were able to identify the so-called vacancy band that characterizes the ordering of the vacancies. This together with the band structure results indicates the important role of the very large inter-Nb-4d hybridization for the formation of the ordered vacancies and the high thermal stability of the ordered structure of niobium monoxide.
\end{abstract}

The transition metal monoxide $\mathrm{NbO}$ is special. While many transition metal monoxides adopt the highly dense rocksalt crystal structure, $\mathrm{NbO}$, synthesized already more than 150 years ago [1], crystallizes in a structure in which $25 \%$ of the $\mathrm{Nb}$ and $25 \%$ of the $\mathrm{O}$ ions are removed from the rocksalt lattice. The $\mathrm{Nb}$ and $\mathrm{O}$ vacancies are ordered and there are no additional distortions of the lattice [2 4], see Fig. 1. For clarity, the hypothetical rocksalt and the actual vacancy-ordered crystal structure of niobium monoxide will be referred to as $\mathrm{Nb}_{4} \mathrm{O}_{4}$ and $\mathrm{Nb}_{3} \mathrm{O}_{3}$, respectively. Adding to the astonishment, the vacancy order is robust and crystal structure remains stable up until $2213 \mathrm{~K}$, the melting point of niobium monoxide 3, 5,8 .

While the formation of defects or vacancies in either the cation or anion sites is in itself not a rare phenomenon for transition metal (TM) oxides [9 11], the precise and robust ordering of the vacancies in niobium monoxide give perhaps credit to efforts that treat its crystal structure not so much as a defect problem but as a three-dimensional network of corner-sharing condensates of $\mathrm{Nb}_{6} \mathrm{O}_{12}$ clusters. This is motivated by the analogy with the $\mathrm{TM}_{6} X_{12}$ clusters that form Chevrel phases ( $X=$ halogen, chalcogen or pnictide) 10, 12, 13. Fig. 1 (right) gives an illustration of such a building block.

Density functional based band structure calculations have been performed [12, 14 -16, to explain the properties of niobium monoxide [17-20] and it was found that the $\mathrm{Nb}_{3} \mathrm{O}_{3}$ structure is more stable than the $\mathrm{Nb}_{4} \mathrm{O}_{4}$ by about $1 \mathrm{eV}$ per $\mathrm{NbO}$ unit [15, 16. Our objective here is to test experimentally the accuracy of band structure calculations and to identify the bands in the measured spectra that are characteristic for $\mathrm{Nb}_{3} \mathrm{O}_{3}$, thereby proving that the vacancies are ordered and that band formation is an essential ingredient for the formation of vacancies in niobium monoxide.

Angle-integrated photoelectron spectroscopy measure-
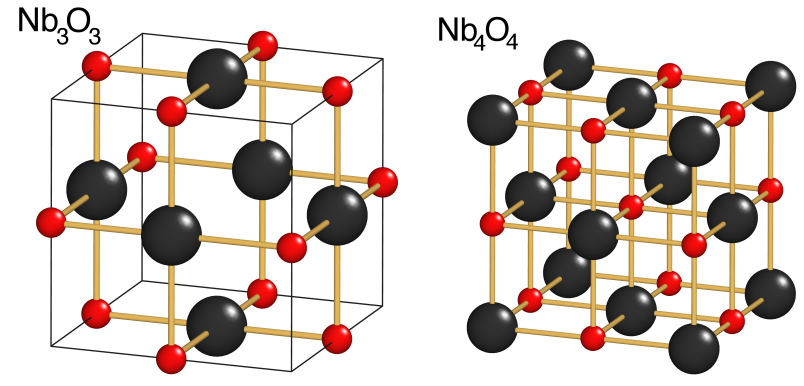

FIG. 1. Crystal structure of the standard rocksalt lattice (left) and the long-range vacancy-ordered rocksalt lattice with one quarter of the atoms missing from both sublattices (right). The large and small spheres depict niobium and oxygen atoms, respectively. Niobium monoxide in the hypothetical rocksalt structure is labeled as $\mathrm{Nb}_{4} \mathrm{O}_{4}$ and in the real vacancyordered structure as $\mathrm{Nb}_{3} \mathrm{O}_{3}$.

ments have been carried out at three facilities: (1) the Dragon beamline $11 \mathrm{~A}$ at the National Synchrotron Radiation Research Center (NSRRC) in Taiwan with the energy of the soft x-rays set to $h \nu=700 \mathrm{eV},(2)$ at the Max-Planck-Institute photoemission facility in Dresden having a monochromatized $\mathrm{Al}-K_{\alpha} h \nu=1486.6 \mathrm{eV}$ x-ray source, and (3) at the Max-Planck-NSRRC hard x-ray photoelectron spectrosopy (HAXPES) end-station [21] at the Taiwan undulator beamline BL12XU of SPring-8 in Japan with the photon energy set to $h \nu=6.5 \mathrm{keV}$. The photoemission facilities in Taiwan (1) and Japan (3) were equipped with MB Scientific A-1 electron energy analyzers, and the one in Dresden (2) with a VG Scienta R3000. Angle-resolved photoelectron spectroscopy (ARPES) was performed at the (1) NSRRC Dragon beamline 11A with the photon energies varied between 90 and $185 \mathrm{eV}$ in order to cover the fifth Brillouin zone of the niobium monoxide. Single crystals of niobium monoxide were grown by 


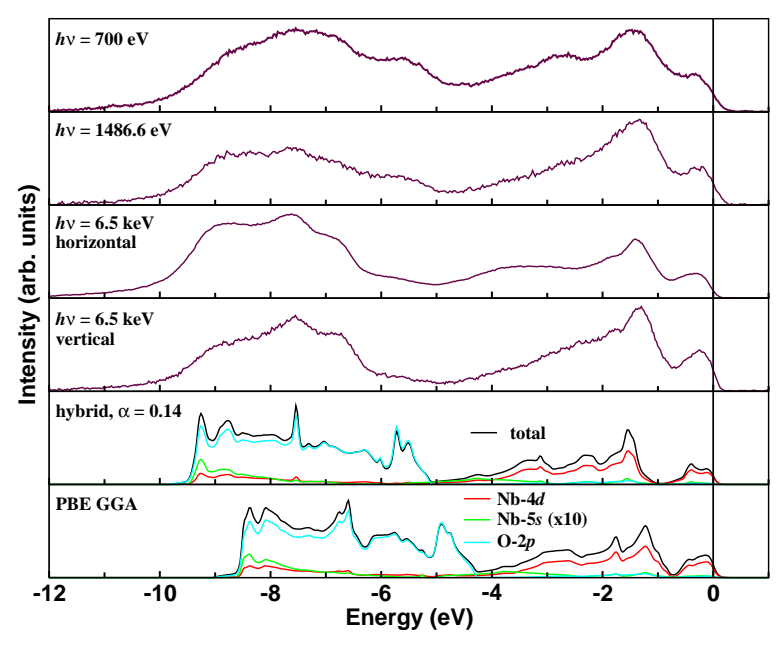

FIG. 2. Valence band spectra of niobium monoxide taken with photon energies $h \nu=700 \mathrm{eV}, 1486.6 \mathrm{eV}$ and $6.5 \mathrm{keV}$ together with the total and orbital projected partial density of states calculated using the hybrid functional and PBE-GGA exchange-correlation functional.

the traveling-solvent floating zone method. The samples were ex-situ aligned using a Laue camera and cleaved in-situ under ultra-high vacuum conditions. The [001] crystal direction is set at normal emission to the electron analyzer lens opening.

The electronic structure calculations were performed using WIEN2k, an augmented plane wave plus local orbitals program [22]. Two kinds of parametrization of the exchange-correlation potential were employed: the Perdew, Burke and Ernzerhof (PBE) parametrization within the generalized gradient approximation (GGA) [23] and a screened hybrid functional for all the electrons [24 26]. The screened hybrid functional $\left(E_{x c}^{\text {hybrid }}\right)$ was constructed such that a part $(\alpha)$ of the semi local PBEGGA exchange $\left(E_{x}^{\mathrm{GGA}}\right)$ was replaced by the short-range part of the Hartree-Fock exchange $\left(E_{x}^{\mathrm{HF}}\right)$ according to,

$$
E_{x c}^{\mathrm{hybrid}}=\alpha E_{x}^{\mathrm{HF}}+(1-\alpha) E_{x}^{\mathrm{GGA}}+E_{c}^{\mathrm{GGA}}
$$

where $E_{c}^{\mathrm{GGA}}$ is the correlation energy. We varied $\alpha$ from 0 to 0.3 , and the best fit was found for $\alpha=0.14$ as will be shown below. The Brillouin zone was sampled by a well converged mesh of $5000 k$-points in the full zone. The experimental lattice constant $a=4.21 \AA$ has been used throughout [4].

Figure 2 displays the valence band (VB) photoemission spectra of niobium monoxide taken with $h \nu=700 \mathrm{eV}$, $1486.6 \mathrm{eV}$ and $6.5 \mathrm{keV}$ photon energies. The $700 \mathrm{eV}$ spectrum was taken at room temperature with an overall energy resolution of $0.7 \mathrm{eV}$, the $1486.6 \mathrm{eV}$ spectrum at room temperature with $0.4 \mathrm{eV}$ resolution, and the $6.5 \mathrm{keV}$ spectra at $80 \mathrm{~K}$ with $0.17 \mathrm{eV}$ resolution. All spectra are normalized to their integrated intensities after the subtraction of the standard integral background to account for inelastic scattering processes [27]. All the spectra show a clear cutoff at zero energy $\left(E_{\mathrm{F}}\right.$, Fermi level), consistent with the system being a good metal [17, 28, 30. All spectra are comparable to one another in terms of the total band width and peak positions, affirming their intrinsic nature. The intensities of the spectral features vary with photon energy, reflecting the photon energy dependence of the photo-ionization cross-sections of the atomic orbitals contributing to the $\mathrm{VB}$ : the Nb- $4 d, \mathrm{O}-2 p$ and also the Nb-5s 31 34].

The $6.5 \mathrm{keV}$ spectra were taken with two different geometries. In the so-called horizontal geometry, the electron energy analyzer was mounted horizontally and parallel to the electrical field vector of the photon beam, while in the vertical geometry, it is perpendicular to the electrical field vector and the Poynting vector of the beam [21]. The spectral intensities depend strongly on the polarization of the light and is given by the so-called $\beta$-asymmetry parameter of the photo-ionization crosssections of the various atomic shells involved [31 34. In particular, it has been shown experimentally [21], that the $s$ contribution to the spectra is substantially reduced in the vertical geometry. The differences in the $6.5 \mathrm{keV}$ spectra for the two geometries thus gives an indication for the energy distribution of the Nb- $5 s$ states.

VB photoemission spectra have been reported previously for very low energy incident photons with $h \nu=30$, 68 and $83.8 \mathrm{eV}$ [18, 19]. Considering the enormous energy difference of the incident photons with the present experiments, the peaks around $-1.5 \mathrm{eV}$ and $-7 \mathrm{eV}$ energies, along with a shoulder around $-5.5 \mathrm{eV}$ are qualitatively similar with those earlier reports [18, 19]. Yet, we are now able to discern additional features due to the improved energy resolution and larger variation of the photo-ionization cross-sections, which help us to present a more precise analysis of the electronic structure of niobium monoxide as discussed below.

We begin by comparing the experimental VB spectra with the calculated density of states (DOS) for $\mathrm{Nb}_{3} \mathrm{O}_{3}$ using the PBE-GGA exchange-correlation functional (Fig. 2, bottom panel). To facilitate an easy comparison, the calculated DOS was multiplied with the Fermi function. On the first glance, the general features of the experimental data seem to be reproduced by these calculations, with the spectral weight closest to the Fermi level $(0$ to $-4 \mathrm{eV}$ ) originating mainly from $\mathrm{Nb}-4 d$ states, while the spectral weight at deeper energies $(-5$ to $-10 \mathrm{eV}$ ) are an admixture of $\mathrm{Nb}-4 d$ and $\mathrm{O}-2 p$ states. On closer inspection, certain discrepancies emerge: first and foremost, the width of the measured spectra $(\sim 10 \mathrm{eV})$ is larger than the calculations $(\sim 8.5 \mathrm{eV})$, thereby reducing the separation between the centers of the Nb- $4 d$ and $\mathrm{O}-2 p$ derived spectral weight. As a consequence, the shoulder around $-5.5 \mathrm{eV}$ in the experiment is seen at $4.8 \mathrm{eV}$ in the calculated DOS. Additionally, the Nb- $4 d$ derived peak at $-1.5 \mathrm{eV}$ energy in the experimental spec- 


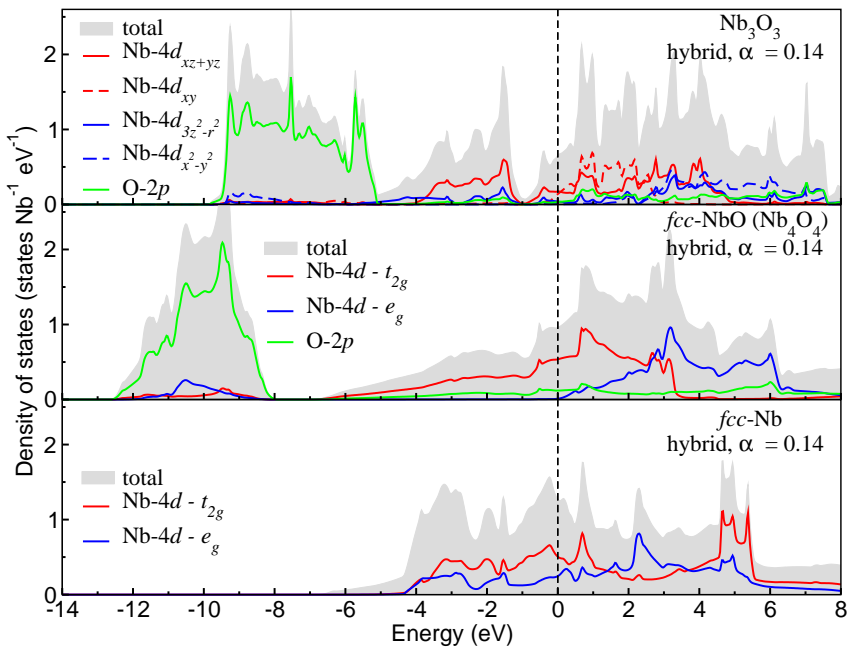

FIG. 3. Total and projected density of states (DOS) for $\mathrm{Nb}_{3} \mathrm{O}_{3}$ (top panel) and the hypothetical $f c c-\mathrm{Nb}_{4} \mathrm{O}_{4}$ (middle panel) using the screened hybrid functional with $\alpha=0.14$. The bottom panel shows the DOS for $\mathrm{Nb}$ metal in the $f c c$ crystal structure. A lattice constant of $a=4.21 \AA$ has been used for all three calculations.

tra is shifted slightly closer to the Fermi level in the calculations. Such discrepancies are reminiscent of spurious self-interaction effects not sufficiently accounted for in conventional DFT.

An approach to improve the orbital energies is to make use of hybrid functionals where an admixture of the exact Hartree-Fock exchange to conventional DFT functionals is incorporated [24, 25]. We therefore have carried out such calculations by also varying the mixing parameter $\alpha$ (see Eq. 1) from 0 to 0.30 , applied not only to the Nb$4 d$ states, but to all electrons in the system. The optimal mixing parameter for $\mathrm{Nb}_{3} \mathrm{O}_{3}$ turned out to be 0.14 , smaller than the standard value of 0.25 generally used for semiconducting or insulating $3 d$ TM-oxides, and perhaps not inconsistent considering the fact that niobium monoxide is a $4 d$ system and a good metal too. The resulting Nb-4d, O- $2 p$ and Nb-5s partial-DOS are plotted in Fig. 2. The width of the VB using hybrid functional is more extended than that of PBE-GGA and agrees better with the experimental spectra. The O- $2 p$ derived shoulder around $-5.5 \mathrm{eV}$, the Nb-4d peak around $-1.5 \mathrm{eV}$ and the van-Hove-like peak around $-7.5 \mathrm{eV}$ are precisely reproduced in our calculations. The hybrid-functional approach thus provides a highly accurate description of the valence band spectrum.

It should be noted here that the presence of defectfree $f c c-\mathrm{Nb}_{4} \mathrm{O}_{4}$ was previously already deemed hypothetical based on the finding that the $\mathrm{Nb}_{3} \mathrm{O}_{3}$ structure is more stable than the $\mathrm{Nb}_{4} \mathrm{O}_{4}$ by about $1 \mathrm{eV}$ per $\mathrm{NbO}$ unit [15, 16]. Also, the comparison of the DOS to the then available spectroscopic data supported this notion [14, 18]. To confirm that such is still the case even when considering self-interaction corrections, we performed hybrid functional calculations for $\mathrm{Nb}_{4} \mathrm{O}_{4}$ as well, with the same lattice constant $a=4.21 \AA$ as $\mathrm{Nb}_{3} \mathrm{O}_{3}$. The resulting total and projected DOS are presented in Fig. 3: indeed the DOS of $\mathrm{Nb}_{4} \mathrm{O}_{4}$ (middle panel) are very different from that of $\mathrm{Nb}_{3} \mathrm{O}_{3}$ or to our experimental VB spectra (Fig. 2), thus confirming the hypothetical nature of $f c c-\mathrm{Nb}_{4} \mathrm{O}_{4}$.

One immediate striking difference between the DOS of $\mathrm{Nb}_{4} \mathrm{O}_{4}$ and that of $\mathrm{Nb}_{3} \mathrm{O}_{3}$ is the much larger energy spread of the occupied DOS of the $\mathrm{Nb}_{4} \mathrm{O}_{4}$, i.e. extending to $-12.5 \mathrm{eV}$ energy, while the DOS of $\mathrm{Nb}_{3} \mathrm{O}_{3}$ ends already at $-10 \mathrm{eV}$. The fact that the $\mathrm{O} 2 p$ derived states in $\mathrm{Nb}_{4} \mathrm{O}_{4}$ extend to such a deep energy and become separated with a gap from the $\mathrm{Nb} 5 d$ derived states reflects the higher Madelung potential in the denser $\mathrm{Nb}_{4} \mathrm{O}_{4}$ structure. Therefore, the smaller Madelung energy of $\mathrm{Nb}_{3} \mathrm{O}_{3}$ must be (over)compensated by another electronic mechanism for the structure to be more stable.

The top and middle panels of Fig. 3 show a break down of the Nb- $4 d$ orbitals contributing to the VB. One can clearly observe that the $e_{g}$ bands of the $\mathrm{Nb}_{4} \mathrm{O}_{4}$ structure

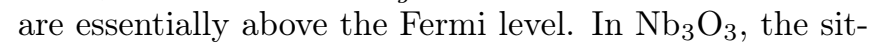
uation is quite different: part of the $e_{g}$ band, namely the $4 d_{3 z^{2}-r^{2}}$, is now extending well below the Fermi level. This constitutes a gain in the formation energy and its origin has been discussed in the past in terms of a local cluster [10, 12, 35]. In $\mathrm{Nb}_{4} \mathrm{O}_{4}$, each $\mathrm{Nb}$ is octahedrally coordinated by six $\mathrm{O}$ ions and the hybridization between them lead to a splitting of the Nb- $4 d$ levels into $t_{2 g}$ and $e_{g}$ states. Since the $\pi$-bonding for the $t_{2 g}$ is significantly smaller than the $\sigma$-bonding for the $e_{g}$, the lowest Nb- $4 d$ states are derived from the $t_{2 g}$ states. With the $\mathrm{Nb}$ having the formal $2+$ valence and thus the $4 d^{3}$ configuration, the occupied states are then made of only the $t_{2 g}$ while the $e_{g}$ remain essentially unoccupied, see middle panel of Fig. 3. In $\mathrm{Nb}_{3} \mathrm{O}_{3}$, the $\mathrm{Nb}$ is locally square planar coordinated by four $\mathrm{O}$ ions. The lack of "apical" oxygens in this coordination makes that there is no $\sigma$-bond for the $4 d_{3 z^{2}-r^{2}}$ orbital so that this state does not get pushed up in energy by the O- $2 p$. Together with the large inter- $4 d$ hybridization, the $4 d_{3 z^{2}-r^{2}}$ can develop bands, part of which is low enough in energy to become occupied [1012, 16, 35, 36, see top panel of Fig. 3. Obviously, with the $4 d_{3 z^{2}-r^{2}}$ becoming partially occupied, the $t_{2 g}$ should move up somewhat to conserve the number of electrons, but apparently this does not cost too much energy, so that in the end there is a net gain of $1 \mathrm{eV}$ per $\mathrm{NbO}$ unit [15, 16] for $\mathrm{Nb}_{3} \mathrm{O}_{3}$ in comparison to $\mathrm{Nb}_{4} \mathrm{O}_{4}$.

It is important to note that the inter- $4 d$ hybridization is quite large. This can be illustrated by calculating the band structure of $f c c \mathrm{Nb}$ metal with the same lattice constant as the hypothetical $f c c-\mathrm{Nb}_{4} \mathrm{O}_{4}$. The results are presented in the bottom panel of Fig. 3. One can observe that both the Nb- $4 d t_{2 g}$ and $e_{g}$ bands have width of roughly $9 \mathrm{eV}$. Such a width implies that correlation effects will not have a chance to stabilize a magnetic or 


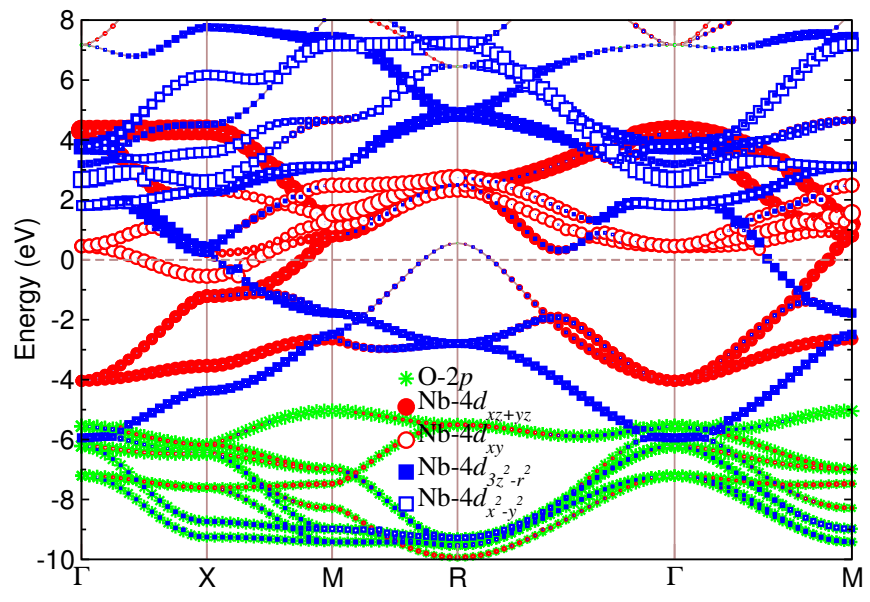

FIG. 4. Calculated band structure including orbital character for $\mathrm{Nb}_{3} \mathrm{O}_{3}$ using the screened hybrid functional with $\alpha=0.14$.

insulating states, and that a non-magnetic metallic solution for the ground state of $\mathrm{Nb}_{4} \mathrm{O}_{4}$ and also $\mathrm{Nb}_{3} \mathrm{O}_{3}$ will be preferred. It is also precisely this large inter- $4 d$ band width which allows for the partial filling of the $4 d_{3 z^{2}-r^{2}}$ band once it is not pushed up anymore to high energies by the $\mathrm{O}-2 p$ as the $\mathrm{Nb}$ is in a local square planar symmetry in the $\mathrm{Nb}_{3} \mathrm{O}_{3}$ structure.

We would like to point out that the situation in $3 d$ transition metal monoxides is quite different. We have calculated that, for example, the inter-3d band width for $f c c \mathrm{~V}$ with the $\mathrm{VO}$ lattice constant of $a=4.073 \AA$ is about $4 \mathrm{eV}$ and for $f c c \mathrm{Ni}$ with the $\mathrm{NiO}$ lattice constant of $a=4.176 \AA$ is about $2 \mathrm{eV}$ (see Supplemental Material [37]). With such smaller one-electron band widths, electron correlation effects will manifest with the result that magnetic and insulating solutions can (and are) realized, thereby reducing tremendously the effective or 'ARPES' dispersions of the $3 d$ derived bands. Thus for $3 d$ oxides one may very well need quite different energy considerations to explain the formation of defects which then also will have a more localized nature. A band structure approach alone may not be adequate.

With the inter- $4 d$ band width in niobium monoxide being very large, the interaction between the vacancies will also be long ranged, so that ordering of the vacancies can be readily expected and consequently, 'vacancy' bands are formed [14, 16]. To illustrate this, we display in Fig. 4 the $k$-dependent band structure where we have put labels for the orbital character of the various bands. These results are very similar to earlier studies [14, 16, 38, 39] but with the difference that we used hybrid functionals with $\alpha=0.14$ in order to have the best agreement with the experiment (Fig. 2). One can clearly see that below the Fermi level there are heavily dispersing bands with a predominantly Nb- $4 d_{3 z^{2}-r^{2}}$ character. These are the 'vacancy' bands of niobium monoxide [14, 16].

Our next task is to show experimentally that these
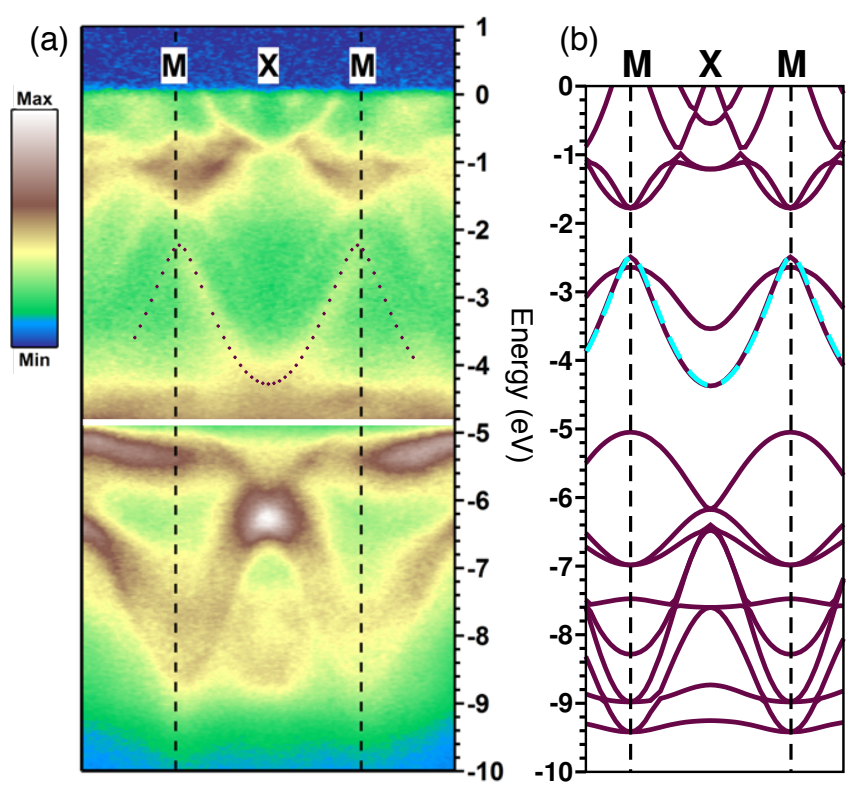

FIG. 5. (a) Experimental angle-resolved photoelectron spectroscopy (ARPES) images along $M-X-M$ with $h \nu=168 \mathrm{eV}$. (b) Calculated band structure along $M-X-M$ using the screened hybrid functional with $\alpha=0.14$. The 'vacancy-band' is highlighted using dotted/dashed lines in both panels.

vacancy bands indeed exist, and by that, to justify the band structure approach for the understanding of the vacancy formation and ordering of the vacancies in niobium monoxide. Fig. 5(a) depicts the experimental intensity image of the ARPES spectrum of niobium monoxide along the $M-X-M$ path measured at $h \nu=168 \mathrm{eV}$ (see Supplemental Material for further experimental details [37]). The dispersing features in the image can be grouped into three energy sections: (i) the electron and hole pockets spanning from $E_{\mathrm{F}}$ to $-1.4 \mathrm{eV}$; (ii) one medium and one strongly dispersive band spanning $-2 \mathrm{eV}$ to $-4.5 \mathrm{eV}$; (iii) a dense set of oxygen bands below $-5 \mathrm{eV}$. Fig. 5(b) displays the calculated band structure along the same $M-X-M$ path. We can observe good general agreement between the measured and calculated band structure. In all the three energy sections mentioned above the band energy positions and dispersions are well reproduced. Of utmost relevance to our work is the middle section where we can observe a dispersive band which we have highlighted using dotted/dashed lines in Fig. 5(a) and (b). This is the Nb- $4 d_{3 z^{2}-r^{2}}$ 'vacancy' band as predicted by the band structure calculations.

To summarize, we have performed angle-integrated and angle-resolved photoelectron spectroscopy measurements on niobium monoxide to investigate its electronic structure and the relationship with the vacancy ordered crystal structure. We have established that band theory provides a good approach and can describe the valence band features accurately by taking into account also corrections for self-interaction effects. The large inter-Nb-4d 
band width plays an important role in the stabilization and ordering of the vacancies and this is clearly demonstrated by the identification of the so-called 'vacancy' band in the experimental angle-resolved spectra.

We thank A. A. Tsirlin for fruitful discussions and T. Mende for skillful technical assistance. D.K. gratefully acknowledges financial support from the Deutsche Forschungsgemeinschaft through project FOR 1346.

* Hao.Tjeng@cpfs.mpg.de

† Deepa.Kasinathan@cpfs.mpg.de

[1] H. Rose, "Ueber das Niob," Pogg. Ann. 104, 310 (1858).

[2] G. Brauer, "Die Oxyde des Niobs," Z. anorg. allg. Chem. 248, 1 (1941).

[3] G. Andersson and A. Magnéli, "Note on the Crystal Structure of Niobium Monoxide," Acta Chem. Scand. 11, 1065 (1957).

[4] A. L. Bowman, T. C. Wallace, J. L. Yarnell, and R. G. Wenzel, "The crystal structure of niobium monoxide," Acta. Crystallogr. 21, 843 (1966).

[5] O. P. Kolchin and N. V. Sumarokova, "The melting point and other properties of the lower oxides of niobium," Atomnaya Energiya 10, 168 (1961).

[6] A. Taylor and N. J. Doyle, "The thermal expansion of titanium, vanadium and niobium monoxides," J. Appl. Crystallogr. 4, 103 (1971).

[7] A. Taylor and N. J. Doyle, "Compressibilities and Grüneisen constants of the monoxides of titanium, vanadium and niobium," J. Appl. Crystallogr. 4, 109 (1971).

[8] T. B. Reed, E. R. Pollard, L. E. Lonney, R. E. Loehman, and J. M. Honig, "Niobium monoxide," in Inorganic Syntheses, Volume 14, edited by A. Wold and J. K. Ruff (John Wiley \& Sons, Inc., 1973) pp. 131-134.

[9] A. D. Wadsley, "Inorganic nonstoichiometric compounds," in Nonstoichiometric Compounds, edited by L. Mandelcorn (Academic Press, New York and London, 1964) pp. 98-209.

[10] H. Schäfer and H. G. von Schnering, "Metall-MetallBindungen bei niederen Halogeniden, Oxyden und Oxydhalogeniden schwerer Übergangsmetalle Thermochemische und strukturelle Prinzipien," Angew. Chem. 76, 833 (1964).

[11] J. K. Burdett and J. F. Mitchell, "Nonstoichiometry in early transition metal compounds with the rocksalt structure," Prog. Solid St. Chem. 23, 131 (1995).

[12] O. K. Andersen and S. Satpathy, "Calculation of the electronic bandstructure for the $3 d$-monoxides and the vacancy compound $\mathrm{Nb}_{3} \mathrm{O}_{3}$," in Basic properties of binary oxides, edited by A. Dominguez Rodriguez, J. Casting, and R. Marquez (Servicio de Publicationes de la Universidad de Sevilla (Serie Ciencias), Sevilla, 1984) pp. 21-42.

[13] R. Chevrel and M. Sergent, "From three-dimensional to one-dimensional cluster $\mathrm{Mo}_{6}$ chalcogenides," in Crystal Chemistry and Properties of Materials with Quasi-OneDimensional Structures: A Chemical and Physical Synthetic Approach, edited by Jean Rouxel (Springer Netherlands, Dordrecht, 1986) pp. 315-373.

[14] E. Wimmer, K. Schwarz, R. Podloucky, P. Herzig, and A. Neckel, "The effect of vacancies on the electronic struc- ture of NbO," J. Phys. Chem. Solids 43, 439 (1982).

[15] K. H. Schwarz, "Band structure and non-stoichiometry of metallic oxides," in Basic properties of binary oxides, edited by A. Dominguez Rodriguez, J. Casting, and R. Marquez (Servicio de Publicationes de la Universidad de Sevilla (Serie Ciencias), Sevilla, 1984) pp. 43-56.

[16] W. W. Schulz and R. M. Wentzcovitch, "Electronic band structure and bonding in $\mathrm{Nb}_{3} \mathrm{O}_{3}$," Phys. Rev. B 48, 16986 (1993).

[17] A. M. Okaz and P. H. Keesom, "Specific heat and magnetization of the superconducting monoxides: $\mathrm{NbO}$ and TiO," Phys. Rev. B 12, 4917 (1975).

[18] J. M. Honig, A. P. B. Sinha, W. E. Wahnsiedler, and H. Kuwamoto, "Studies of the Band Structure of NbO by X-Ray Photoelectron Spectroscopy," Phys. Stat. Sol. B73, 651 (1976).

[19] M. Erbudak, V. A. Gubanov, and E. Z. Kurmaev, "The electronic structure of NbO: Theory and experiment," J. Phys. Chem. Solids 39, 1157 (1978).

[20] W. W. Schulz, L. Forro, C. Kendziora, R. Wentzcovitch, D. Mandrus, L. Mihaly, and P. B. Allen, "Band structure and electronic transport properties of the superconductor NbO," Phys. Rev. B 46, 46 (1992).

[21] J. Weinen, T. C. Koethe, C. F. Chang, S. Agrestini, D. Kasinathan, Y. F. Liao, H. Fujiwara, C. SchüßlerLangeheine, F. Strigari, T. Haupricht, G. Panaccione, F. Offi, G. Monaco, Huotari. H., K.-D. Tsuei, and L. H. Tjeng, "Polarization dependent hard x-ray photoemission experiments for solids: Efficiency and limits for unraveling the orbital character of the valence band," J. Elec. Spectroscopy and Related Phenomena 198, 6 (2015).

[22] P. Blaha, K. Schwarz, G. K. H. Madsen, D. Kvasnicka, and J. Luitz, WIEN2k, An Augmented Plane Wave + Local Orbitals Program for Calculating Crystal Properties (Karlheinz Schwarz, Techn. Universität Wien, Austria, 2001).

[23] J. P. Perdew, K. Burke, and M. Ernzerhof, "Generalized gradient approximation made simple," Phys. Rev. Lett. 77, 3865 (1996).

[24] J. Heyd, G. E. Scuseria, and M. Ernzerhof, "Hybrid functionals based on a screened Coulomb potential," J. Chem. Phys. 118, 8207 (2003); 124, 219906 (2006).

[25] J. Heyd and G. E. Scuseria, "Efficient hybrid density functional calculations in solids: assessment of the HeydScuseria-Ernzerhof screened Coulomb hybrid functional," J. Chem. Phys. 121, 1187 (2004).

[26] F. Tran and P. Blaha, "Implementation of screened hybrid functionals based on the Yukawa potential within the LAPW basis set," Phys. Rev. B 83, 235118 (2011).

[27] Stefan Hüfner, "Continuous satellites and plasmon satellites: XPS photoemission in nearly free electron systems," in Photoelectron Spectroscopy: Principles and Applications (Springer Berlin Heidelberg, 2003) pp. 173-209.

[28] J. A. Roberson and R. A. Rapp, "Electrical properties of $\mathrm{NbO}$ and $\mathrm{NbO}_{2}$," J. Phys. Chem. Solids 30, 1119 (1969).

[29] J. K. Hulm, C. K. Jones, R. A. Hein, and J. W. Gibson, "Superconductivity in the $\mathrm{TiO}$ and $\mathrm{NbO}$ systems," J. Low Temp. Phys. 7, 291 (1972).

[30] J. M. Honig, W. E. Wahnsiedler, and P. C. Eklund, "Electrical properties of NbO in high magnetic fields," J. Solid State Chem. 6, 203 (1973).

[31] J. Yeh and I. Lindau, "Atomic subshell photoionization cross sections and asymmetry parameters: $1 \leq \mathrm{Z} \leq 103$," Atomic Data and Nuclear Tables 32, 1 (1985). 
[32] M. B. Trzhaskovskaya, V. I. Nefedov, and V. G. Yarzhemsky, "Photoelectron angular distribution parameters for elements $\mathrm{Z}=1$ to $\mathrm{Z}=54$ in the photoelectron energy range 100-5000 eV," Atomic Data and Nuclear Data Tables 77, 97 (2001).

[33] M. B. Trzhaskovskaya, V. I. Nefedov, and V. G. Yarzhemsky, "Photoelectron angular distribution parameters for elements $\mathrm{Z}=55$ to $\mathrm{Z}=100$ in the photoelectron energy range 100-5000 eV," Atomic Data and Nuclear Data Tables 82, 257 (2002).

[34] M. B. Trzhaskovskaya, V. K. Nikulin, V. I. Nefedov, and V. G. Yarzhemsky, "Non-dipole second order parameters of the photoelectron angular distribution for elements $\mathrm{Z}$ $=1-100$ in the photoelectron energy range $1-10 \mathrm{keV}$," Atomic Data and Nuclear Data Tables 92, 245 (2006).

[35] J. K. Burdett and T. Hughbanks, "NbO and TiO: Struc- tural and Electronic Stability of Structures Derived from Rock Salt," J. Am. Chem. Soc. 106, 3101 (1984).

[36] J. K. Burdett and J. F. Mitchell, "Pair Potentials and the Ordered Defect Structure of NbO," Inorg. Chem. 32, 5004 (1993).

[37] See Supplemental Material at http://link.aps.org/supplemental/10.1103/PhysRevLett.000.000000 for details about the ARPES measurement and additional density of states.

[38] Y. Kubo, S. Wakoh, and K. Schwarz, "Theoretical Momentum Distributions in $\mathrm{Nb}_{3} \mathrm{O}_{3}$," J. Phys. Soc. Japan 55, 1266 (1986).

[39] H. Aoki, Y. Asada, T. Hatano, K. Ogawa, A. Yanase, and M. Koiwa, "Fermi surface of NbO," J. Low. Temp. Phys. 81, 19 (1990).

\section{Supplemental Material}

\section{(1) INTER-TRANSITION-METAL $d$ HYBRIDIZATION IN ROCKSALT OXIDES}

In order to estimate the inter-transition-metal hybridization strength in transition metal $4 d$ and $3 d$ monoxides oxides with the rocksalt crystal structure, we calculate the band structure of fcc $\mathrm{NbO} / \mathrm{VO} / \mathrm{NiO}$ and fcc $\mathrm{Nb} / \mathrm{V} / \mathrm{Ni}$ with the same lattice constant as $\mathrm{NbO} / \mathrm{VO} / \mathrm{NiO}$, respectively. See Fig. S1, which also include the $e_{g}$ and $t_{2 g}$ projections.

\section{(2) ARPES: MOMENTUM SPACE MAPPING}

The schematics of the momentum space mapping for the niobium monoxide ARPES experiment is depicted in
Fig. S2. The sample is oriented with the [001] surface normal directed to the electron energy analyzer. An inner potential $V_{0}=9 \mathrm{eV}$ has been used based on the extremal behavior observed in the energy dispersions of various $\mathrm{Nb}$ and $\mathrm{O}$ derived spectral features as function of photon energy. A photon energy interval of 90 to $185 \mathrm{eV}$ allows for a full coverage of the fifth Brillouin zone of niobium monoxide. 

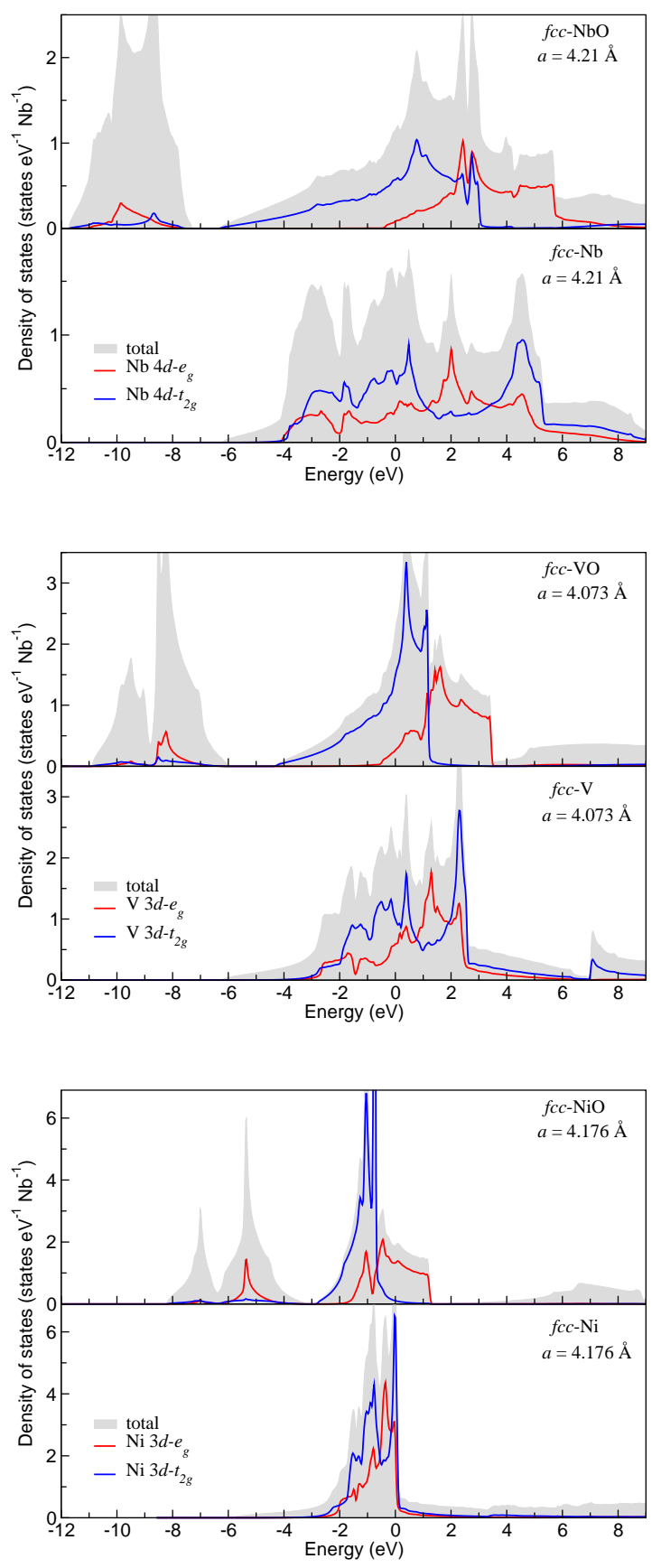

FIG. S1. Total and $d$-projected density of states for fcc NbO, $\mathrm{Nb}, \mathrm{VO}, \mathrm{V}, \mathrm{NiO}$ and $\mathrm{Ni}$. The calculations were performed using the WIEN2k code and the Perdew, Burke and Ernzerhof (PBE) parametrization within the generalized gradient approximation (GGA). 


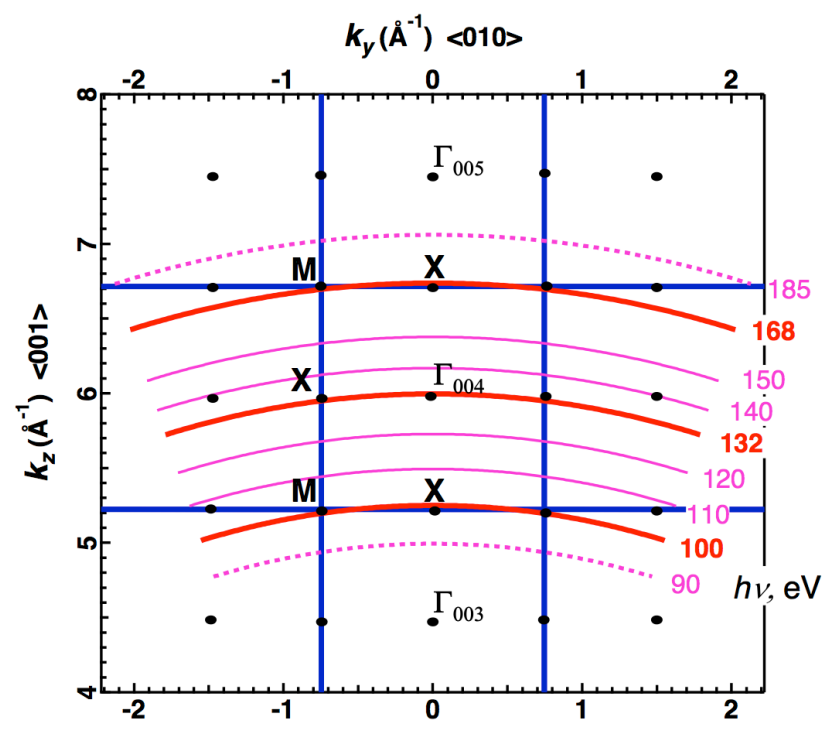

FIG. S2. Schematics of the experimentally probed cuts in the momentum space $k_{y}-k_{z}$ plane with $k_{x}=0$. The cuts are for photoelectrons with zero binding energy with the corresponding photon energies indicated. An inner potential $V_{0}=9 \mathrm{eV}$ has been used. Cuts which pass through the high-symmetry points at normal emission are highlighted by the thick red lines. The electron energy analyzer has a $\pm 18^{\circ}$ angular acceptance with a work function of $3.87 \mathrm{eV}$. 